\title{
Plan de Conservación para la Maquinaria del area de Producción de la Empresa Calzado Selecto Zolinka S.A. de C.V.
}

\section{Juan Carlos Barragan Barajas ${ }^{\text {, J Jorge Armando Ramos Frutos }}$, Juan Pedro Quiñones Reyes ${ }^{c}$, Juan Carlos Magallón Pulido ${ }^{d}$}

${ }^{a}$ Instituto tecnologico de Jiquilpan, Jiquilpan, Mich., Mexico, jcbit@yahoo.com, ${ }^{\mathrm{b}}$ Centro de Innovación Aplicada en Tecnologías Competitivas (CIATEC), León, Gto. Mexico jorgerf1095@gmail.com, c Instituto tecnologico de Jiquilpan, Jiquilpan, Mich., Mexico, pedroq19@yahoo.com.mx, ${ }^{\mathrm{d}}$ Instituto tecnologico de Jiquilpan, Jiquilpan, Mich., Mexico, carlos_mag_20@hotmail.com

\section{Resumen}

La empresa Calzado Selecto Zolinka S.A. de C.V. no cuenta con un Plan de Conservación en los recursos que utiliza en producción. La mayoría de las actividades relacionadas con mantenimiento son de mantenimiento correctivo. Por lo tanto, se pretende diminuir la cantidad de acciones correctivas que incrementen los costos de mantenimiento. El concepto de mantenimiento ha crecido y ha adquirido madurez progresiva para adaptarse a las distintas necesidades y requerimientos de una empresa. La mayoría de las fallas que se presentan en los recursos de la empresa son el resultado de la intensa carga de trabajo. Al inicio de las operaciones sólo se hace mantenimiento correctivo, también conocido como "Mantenimiento de Ruptura". El mantenimiento correctivo se espera a que se presente la falla en la máquina para realizar actividades de conservación. Se pretende realizar una propuesta para que en función de los manuales de operación o recomendaciones del fabricante se inicie a administrar la conservación evitando fallas imprevistas aumentando el rendimiento y prolongar la vida útil de los componentes físicos de la empresa. Para elaborar la propuesta de el plan de conservación de la maquinaria y equipo de la empresa se genera el análisis de criticidad y el análisis del AMEF, esto con el fin de saber de su importancia en el área de producción y a su vez identificar las fallas del diseño de un producto o de un proceso antes de que éstas ocurran.

Palabras clave: Plan de Conservación, maquinaria, Análisis del Modo y Efectos de Falla, Mantenimiento. 


\section{Introducción}

Aplicar mantenimiento correctivo es costoso porque implica demasiado tiempo perdido en el proceso de producción cuando ocurre una falla no prevista. Algunos de los problemas por no tener una gestión adecuada de mantenimiento son: gastos en las reparaciones, problemas con la satisfacción del cliente, probables accidentes que pueden afectar a los operadores, entre otros. Elaborar un plan de mantenimiento preventivo reduciría las afectaciones antes mencionados. Elaborar planes de mantenimiento ayudara a la eficiente gestión del mismo ademas de desarrollar una arma competitiva al incrementar el rendimiento de la empresa.

Contar con un registro de las actividades de conservación es posible programar las acciones preventivas (lubricación, limpieza de abrasivos, cambio de bandas, cojinetes, chumaceras, etc.) Sin interferir con las áreas de producción. El registro también ayuda a prever fallas de la maquinaria para evitar los paros de emergencia en horas de trabajo.

Debido a dicha importancia sobre el mantenimiento preventivo de la maquinaria y equipo, se presenta un plan de conservación. El plan de conservación tiene como finalidad maximizar la disponibilidad y confiabilidad en la maquinaria y equipo del área de producción. Para lograrlo se propone el Mantenimiento Centrado en la Confiabilidad (RCM).

El proyecto se compone de tres capítulos: En el primero, se describen aspectos relevantes de la empresa estos es; sus antecedentes, la situación en la que se encuentra actualmente, sus principales ‘productos, la distribución de la planta y su localización. En el segundo capítulo se presentan los conceptos teóricos necesarios para dar soporte a la investigación y desarrollo del proyecto, tales como, la evolución del mantenimiento, los tipos de mantenimiento, su importancia al aplicarlo. Además, de presentar la técnica del mantenimiento centrado en la confiabilidad (RCM) y el análisis de modo y efecto de fallas (AMEF). Finalmente en el último capítulo se encuentran las actividades realizadas y son: El listado de la maquinaria utilizada para la producción de calzado, el listado de las Herramientas, la clasificación de los equipos de producción dependiendo del nivel de criticidad, el formato utilizado para la realización de las fichas técnicas, y la determinación de fallos funcionales y técnicos y por consiguiente la propuesta de un programa de mantenimiento que satisfaga la necesidades de los recursos físicos por atender. 


\section{Descripción de las Actividades}

En el presente proyecto se tuvo participación de la empresa Calzado Selecto Zolinka y el Instituto Tecnológico de Jiquilpan. Las dos partes trabajaron en conjunto en la gestión de las actividades realizadas para obtener los objetivos del presente. A continuación se presentan las actividades que se realizaron para obtener el Plan de Conservación de la empresa Calzado Selecto Zolinka.

2.1. Listado de la Maquinaria (Inventario Inicial de Maquinaria)

La primera actividad implica realizar una tabla que contiene la maquinaria de producción de la empresa Calzado Selecto Zolinka. Esta lista se realiza elaborando un inventario mostrado en la tabla 1, en la que se especifica la maquinaria, el área a la que pertenece el equipo y sus cantidades.

Tabla 1. Lista de la maquinaria de producción de Calzados Selectos Zolinka.

\begin{tabular}{|l|c|l|}
\hline \multicolumn{1}{|c|}{ Maquina } & Cantidad & \multicolumn{1}{c|}{ Área } \\
\hline Troqueladora de bolsa & 1 & Producción \\
\hline Troqueladora de marca & 1 & Producción \\
\hline Troqueladora de número & 1 & Producción \\
\hline Cabina & 1 & Producción \\
\hline Conformadora de talón & 1 & Producción \\
\hline Doblilladora & 1 & Producción \\
\hline Embarradora de plantilla & 1 & Producción \\
\hline Flejadora & 1 & Empaque \\
\hline Máquina de pegadura & 1 & Producción \\
\hline Máquina de pespunte & 3 & Producción \\
\hline Montadora de talón & 1 & Producción \\
\hline Rebajadora & 2 & Producción \\
\hline Remachadora & 1 & Producción \\
\hline Máquina de ribete & 1 & Producción \\
\hline Suajadora & 2 & Producción \\
\hline Triple arrastre & 1 & Producción \\
\hline Sacadora de correa & 2 & Producción \\
\hline Máquina cortadora de laser & 1 & Producción \\
\hline Suajadora de puente & 1 & Producción \\
\hline Compresora & 1 & Producción \\
\hline Banco de carda & 1 & Producción \\
\hline Flameadora & 1 & Producción \\
\hline
\end{tabular}

Fuente. Elaboracion propia 
Esta lista permite que se genere control en el manejo de los recursos de la empresa. Si existe alguna baja o algún robo se percibe de inmediato porque está contabilizada la maquinaria y equipo de la empresa. De manera similar se realiza este inventario con la herramienta lo que permite ver las fortalezas y debilidades del área de mantenimiento, desarrollando de esta manera el plan mas conveniente para la atención adecuada de los recursos fisicos.

\subsection{Sistema de Codificación de los Equipos}

El siguiente paso se presenta la forma de realizar la codificación de los equipos, La forma en que se codificarán los activos se muestra en la figura 1.

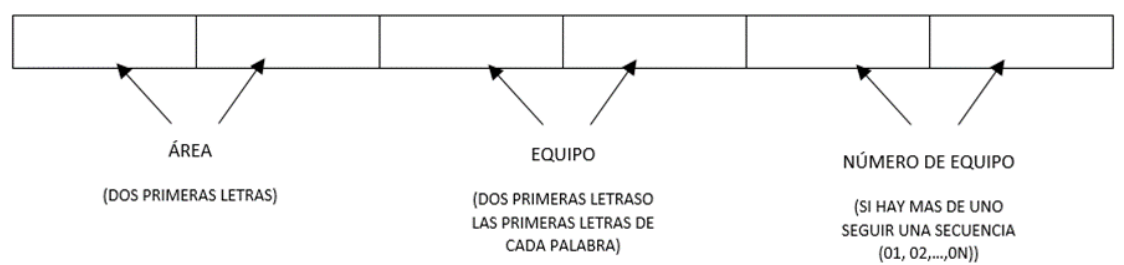

Fig. 1. Sistema de Codificación propuesto

Fuente: (García, 2003)

Como se observa en la Figura 1, el área de la Planta en que está ubicado el equipo estaría definido por dos caracteres alfanuméricos, igual el equipo, y el número consecutivo en relación a la cantidad por dos caracteres numéricos. La tabla 2 muestra los equipos con el código asignado. 
Tabla 2. Codificación de la Lista de Producción

\begin{tabular}{|l|c|c|}
\hline \multicolumn{1}{|c|}{ Equipo } & Sistema & Código \\
\hline Troqueladora de bolsa & Sistema eléctrico e hidráulico & PR-TB-01 \\
\hline Troqueladora de marca & Sistema eléctrico & PR-TM-01 \\
\hline Troqueladora de número & Sistema eléctrico & PR-TN-01 \\
\hline Cabina & Sistema eléctrico & PR-CA-01 \\
\hline Conformadora de talón & Sistema eléctrico y neumático & PR-CT-01 \\
\hline Doblilladora & Sistema eléctrico & PR-DO-01 \\
\hline Embarradora de plantilla & Sistema eléctrico & PR-EP-01 \\
\hline Flejadora & Sistema eléctrico & EM-FL-01 \\
\hline Máquina de pegadura & Sistema eléctrico y neumático & PR-MP-01 \\
\hline Máquina de pespunte & Sistema hidráulico y eléctrico & PR-PE-01 \\
\hline Máquina de pespunte & Sistema hidráulico y eléctrico & PR-PE-02 \\
\hline Máquina de pespunte & Sistema hidráulico y eléctrico & PR-PE-03 \\
\hline Montadora de talón & Sistema eléctrico y neumático & PR-MT-01 \\
\hline Rebajadora & Sistema hidráulico y eléctrico & PR-RE-01 \\
\hline Rebajadora & Sistema hidráulico y eléctrico & PR-RE-02 \\
\hline Remachadora & Sistema mecánico & PR-RM-01 \\
\hline Remachadora & Sistema mecánico & PR-RM-02 \\
\hline Máquina de ribete & Sistema hidráulico y eléctrico & PR-RI-01 \\
\hline Suajadora & Sistema hidráulico y eléctrico & PR-SU-01 \\
\hline Suajadora & Sistema hidráulico y eléctrico & PR-SU-02 \\
\hline Triple arrastre & Sistema hidráulico y eléctrico & PR-TR-01 \\
\hline Sacadora de correa & Sistema eléctrico & PR-SC-01 \\
\hline Sacadora de correa & Sistema eléctrico & PR-SC-02 \\
\hline Máquina cortadora de laser & PR-CL-01 \\
\hline Suajadora de puente & Sistema eléctrico y neumático & PR-CP-01 \\
\hline Compresora & Sistema eléctrico & PR-BC-01 \\
\hline Banco de cardar & Seléctrico & PR-FL-01 \\
\hline Flameadora & Sidráulico y eléctrico & PR-SP-01 \\
\hline
\end{tabular}

Fuente. Elaboracion propia

De esta manera se pueden rastrear con mayor eficiencia los equipos, logrando un mejor control e incrementando la productividad del área de mantenimiento.

\subsection{Análisis de Criticidad}

Los equipos de producción tienen diferentes niveles de importancia en una planta industrial. Dada la limitación de recursos de una empresa para mantener la planta, se debe destinar la mayor parte de esos recursos a los equipos más importantes, logrando resolver en un alto porcentaje la mayoría de los problemas que puedan presentarse en relación a las necesidades de los equipos por atender.

Según el principio de V. Pareto, aproximadamente el $20 \%$ de las causas son responsables del $80 \%$ del efecto total; y el $80 \%$ de las causas restantes solo son responsables del $20 \%$ 
De acuerdo con el libro La Productivdad en el Mantenimeinto Indsutrial de Enrique Douce Villanueva.

Para poder apreciar los diferentes tipos de criticidad véase la tabla 3. Después de analizar cada uno de los equipos utilizados para la producción, con los criterios anteriores se define el nivel de criticidad dentro del área de producción, Se realiza el análisis a cada uno de los recursos a clasificar. Dicha tarea se presenta resumida en la tabla 4.

\subsection{Análisis del Modo y Efectos de Fallo}

Para poder aplicar el Análisis del Modo y Efectos de Fallo (AMEF) es necesario realizar el análisis para cada uno de los equipos, se aplica mediante un proceso continuo para la identificación de las fallas en los equipos antes de que éstas ocurran, por lo que se utilizará una tabla con cinco columnas: la primera columna se refiere al equipo, la segunda al sistema, la tercera al tipo de fallo, la cuarta a la descripción del fallo, la quinta a la descripción del modo de fallo. En la tabla 5 se muestra el AMEF de un solo equipo, dado que la tabla que se genera es muy extensa. En la tabla 5 se muestran los fallos técnico, fallos funcionales, modos de fallo, entre otras columnas que permiten tener un registro de cada equipo y de esa manera reducir los tiempos de conservación. 
Tabla 3. Codificación de la Lista de Producción

\begin{tabular}{|c|c|c|c|c|}
\hline Tipo de equipo & $\begin{array}{l}\text { Seguridad y medio } \\
\text { ambiente }\end{array}$ & Producción & Calidad & $\begin{array}{c}\text { Mantenimient } \\
\mathrm{o}\end{array}$ \\
\hline \multirow[b]{3}{*}{ Critico (A) } & $\begin{array}{c}\text { Pueden originar acciones } \\
\text { muy graves. }\end{array}$ & \multirow{3}{*}{$\begin{array}{l}\text { Su parada } \\
\text { afecta al } \\
\text { plan de } \\
\text { producción }\end{array}$} & $\begin{array}{l}\text { Es clave para } \\
\text { la calidad del } \\
\text { producto }\end{array}$ & $\begin{array}{l}\text { Alto costo de } \\
\text { reparación en } \\
\text { caso de avería }\end{array}$ \\
\hline & $\begin{array}{c}\text { Necesita revisiones } \\
\text { periódicas frecuente } \\
\text { (mensuales) }\end{array}$ & & \multirow[b]{2}{*}{$\begin{array}{l}\text { Es causante } \\
\text { de un alto } \\
\text { porcentaje de } \\
\text { rechazos }\end{array}$} & $\begin{array}{l}\text { Averías muy } \\
\text { frecuentes }\end{array}$ \\
\hline & $\begin{array}{l}\text { Ha producido acciones en } \\
\text { el pasado }\end{array}$ & & & $\begin{array}{c}\text { Consume una } \\
\text { parte } \\
\text { importante de } \\
\text { los recursos de } \\
\text { mantenimient } \\
\text { o (mano de } \\
\text { obra y/o } \\
\text { materiales) }\end{array}$ \\
\hline & $\begin{array}{l}\text { Necesita revisiones } \\
\text { periódicas (anuales) }\end{array}$ & \multirow{2}{*}{$\begin{array}{c}\text { Afecta a la } \\
\text { producción } \\
\text {, pero es } \\
\text { recuperabl } \\
\text { e (no llega } \\
\text { a afectar a } \\
\text { clientes o } \\
\text { plan de } \\
\text { producción } \\
\text { ) } \\
\end{array}$} & \multirow[b]{2}{*}{$\begin{array}{c}\text { Afecta a la } \\
\text { calidad, pero } \\
\text { habitualment } \\
\text { e no es } \\
\text { problemática }\end{array}$} & \multirow[b]{2}{*}{$\begin{array}{l}\text { Costo medio } \\
\text { en } \\
\text { mantenimient } \\
\text { o }\end{array}$} \\
\hline Importante (B) & $\begin{array}{l}\text { Puede ocasionar accidente } \\
\text { grave, pero las } \\
\text { probabilidades son } \\
\text { remotas }\end{array}$ & & & \\
\hline $\begin{array}{l}\text { Prescindible } \\
\text { (C) }\end{array}$ & $\begin{array}{l}\text { Poca influencia en } \\
\text { seguridad }\end{array}$ & $\begin{array}{c}\text { Poca } \\
\text { influencia }\end{array}$ & $\begin{array}{l}\text { No afecta a la } \\
\text { calidad }\end{array}$ & $\begin{array}{c}\text { Bajo costo de } \\
\text { mantenimient } \\
\text { o }\end{array}$ \\
\hline
\end{tabular}

Fuente. Elaboracion propia 
Plan de Conservacion para la Maquinaria del area de Produccion de la Empresa Calzado Selecto Zolinka S.A. de C.V.

Tabla 4. Resúmen del Análisis de Criticidad

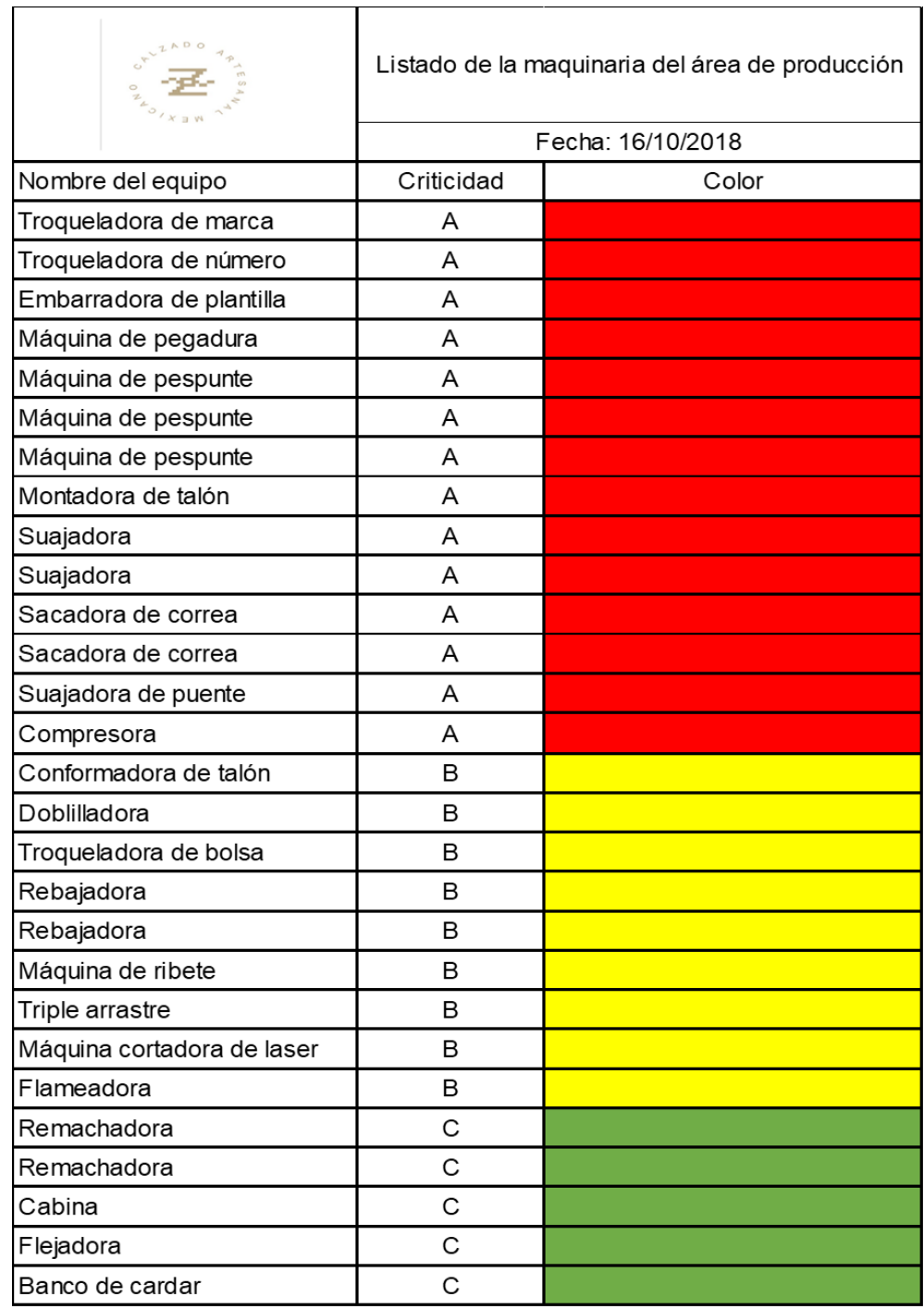

Fuente. Elaboracion propia

Editorial Universitat Politècnica de València 
Tabla 5. Análisis del Modo y Efectos de Fallo

\begin{tabular}{|c|c|c|c|c|}
\hline Equipo & Sistema & $\begin{array}{l}\text { Tipo de } \\
\text { fallo }\end{array}$ & $\begin{array}{l}\text { Descripción de la } \\
\text { falla }\end{array}$ & $\begin{array}{l}\text { Descripción del } \\
\text { modo de fallo }\end{array}$ \\
\hline \multirow{11}{*}{$\begin{array}{c}\text { Troqueladora } \\
\text { de bolsa }\end{array}$} & \multirow{4}{*}{$\begin{array}{l}\text { sistema } \\
\text { eléctrico }\end{array}$} & \multirow{3}{*}{$\begin{array}{l}\text { Fallos } \\
\text { Funcional } \\
\text { es }\end{array}$} & $\begin{array}{c}\text { Fallo en la } \\
\text { alimentación }\end{array}$ & Corto circuito \\
\hline & & & \multirow{2}{*}{$\begin{array}{c}\text { Fallo en la } \\
\text { comunicación del } \\
\text { sistema }\end{array}$} & Falsos contactos \\
\hline & & & & $\begin{array}{c}\text { Bobinas en mal } \\
\text { estado }\end{array}$ \\
\hline & & $\begin{array}{l}\text { Fallos } \\
\text { técnicos }\end{array}$ & $\begin{array}{c}\text { Fallo en la } \\
\text { comunicación del } \\
\text { sistema }\end{array}$ & Falsos contactos \\
\hline & $\begin{array}{l}\text { Sistema } \\
\text { mecánic } \\
\text { o }\end{array}$ & $\begin{array}{l}\text { Fallos } \\
\text { técnicos }\end{array}$ & Ruidos excesivos & $\begin{array}{c}\text { Cojinetes en mal } \\
\text { estado u/o turbina } \\
\text { desgastada }\end{array}$ \\
\hline & \multirow{6}{*}{$\begin{array}{c}\text { Sistema } \\
\text { Hidráulic } \\
\text { o }\end{array}$} & \multirow{4}{*}{$\begin{array}{c}\text { Fallos } \\
\text { Funcional } \\
\text { es }\end{array}$} & \multirow{2}{*}{$\begin{array}{c}\text { Fallo en la Bomba } \\
\text { de aceite }\end{array}$} & Retenes en mal estado \\
\hline & & & & $\begin{array}{l}\text { Mal funcionamiento } \\
\text { de la bomba }\end{array}$ \\
\hline & & & Falta de presión & Falta de aceite \\
\hline & & & Falla en el troquel & Falla de Sensores \\
\hline & & \multirow{2}{*}{$\begin{array}{c}\text { Fallo } \\
\text { Técnicos }\end{array}$} & $\begin{array}{c}\text { Fallo en las } \\
\text { mangueras }\end{array}$ & Fugas de aceite \\
\hline & & & $\begin{array}{c}\text { Fallo en las } \\
\text { válvulas }\end{array}$ & $\begin{array}{c}\text { Obstrucción de las } \\
\text { válvulas }\end{array}$ \\
\hline
\end{tabular}

Fuente. Elaboracion propia

\section{Resultados}

De acuerdo con el análisis anterior se realiza un plan de mantenimiento inicial que se muestra a continuación. Con los resultados del AMEF se percibe que se pueden presentar algunas fallas en el momento de estar llevando a cabo el proceso de producción, las cuales a su vez pueden ser corregidos por el operador. El plan de mantenimiento se realiza para 
cada uno de los recursos con los que cuenta la empresa. En la tabla 6 se muestra el plan de mantenimiento inicial para uno de los recursos de la organización (La máquina denominada doblilladora).

Ahora bien, gracias al análisis del AMEF y al análisis de criticidad se puede saber qué tipo de mantenimiento es necesario aplicar a cada una de la maquinas, por lo tanto, esta propuesta está basada en un plan de mantenimiento preventivo. A continuación, se presentan varias actividades de mantenimiento claves y de gran importancia para conservar la maquinaria en perfecto estado de funcionamiento.

- Actividades de mantenimiento diarias

- Actividades de mantenimiento semanales

- Actividades de mantenimiento mensuales

- Actividades de mantenimiento trimestrales

- $\quad$ Actividades de mantenimiento semestrales

Tabla 6. Plan de Mantenimiento Inicial para la Doblilladora

\begin{tabular}{|c|c|c|c|}
\hline \multirow{6}{*}{ Doblilladora } & $\begin{array}{c}\text { Mantenimiento } \\
\text { diario }\end{array}$ & $\begin{array}{l}\text { Limpieza de } \\
\text { residuos de } \\
\text { pegamento }\end{array}$ & Operador \\
\hline & \multirow{2}{*}{$\begin{array}{c}\text { Mantenimiento } \\
\text { semanal }\end{array}$} & $\begin{array}{c}\text { Lubricación de } \\
\text { engranes del buril }\end{array}$ & $\begin{array}{l}\text { Operador- } \\
\text { mecánico }\end{array}$ \\
\hline & & $\begin{array}{c}\text { Engrasado del } \\
\text { pedal arrancador }\end{array}$ & $\begin{array}{l}\text { Operador- } \\
\text { mecánico }\end{array}$ \\
\hline & \multirow[b]{3}{*}{$\begin{array}{l}\text { Mantenimiento } \\
\text { semestral }\end{array}$} & $\begin{array}{l}\text { Engrasado de } \\
\text { cojinetes del } \\
\text { motor }\end{array}$ & Mecánico \\
\hline & & $\begin{array}{c}\text { Cambio de } \\
\text { banda }\end{array}$ & $\begin{array}{l}\text { Operador- } \\
\text { mecánico }\end{array}$ \\
\hline & & $\begin{array}{c}\text { Limpieza de } \\
\text { abrasivos } \\
\text { acumulados en } \\
\text { partes eléctricas } \\
\text { (interruptores, } \\
\text { resistencias, etc.) }\end{array}$ & Mecánico \\
\hline
\end{tabular}

Fuente. Elaboracion propia

En cada uno de los recursos se hace un plan de mantenimiento inicial. Se pretende aplicar el ciclo de mejora continua en la declaración de las actividades de mantenimiento. 


\section{Conclusiones}

Los equipos e instalaciones de una industria están sometidos a varios tipos de mantenimiento, que pueden ser correctivo, predictivo, preventivo o proactivo, cada uno de estos son aplicables a la maquinaria en la proporción que este lo requiere. La planeación y programación del plan de mantenimiento tiene la finalidad de trazar las actividades o acciones que deben realizarse durante la jornada de trabajo para el mejor desempeño de la maquinaria. Por ende, el mantenimiento debe proporcionar confiabilidad, eficiencia y productividad a la industria dado que los resultados se evalúan en cantidad y calidad de producto.

\section{Agradecimientos}

Los autores agradecen el apoyo a la empresa Calzado Selecto Zolinka y al Instituto Tecnológico de Jiquilpan.

\section{Referencias}

Barreda, S. (2015). Plan de Mantenimiento Centrado en la Confiabilidad Aplicado en la EDAR. Nules -Villavella.

Cuartas, L. (2008). http://www.unalmed.edu.co. Obtenido de unalmed: http://www.unalmed.edu.co/tmp/curso_concurso/area3/QUE_ES_EL_MANTENIMIENTO_MEC ANICO.pdf

Dounce E. (2017) La Productividad en el Mantenimiento Industrial. Ciudad de México, 3ra. Edición.

Ebelling, C. (2005). An Introduction to Reliability and Maintainability Engineering. New York City: Waveland Press Inc.

Esquivel, J. (2009). METODOLOGÍA PARA LA DETECCIÓN Y PREVENCIÓN DE FALLAS EN EQUIPOS INDUSTRIALES DE PRODUCCIÓN. México: Ciudad Universitaria.

García, S. (2003). Organización y Gestión Integral del Mantenimiento. Madrid: Días de Santos.

Gutiérrez, J. (2008). Desarrollo de una metodología de mantenimiento centrado en confiabilidad para líneas de transmisión en alta tensión. Pereira.

Lyonnet, P. (2002). Manual del Ingeniero de Mantenimiento.

Mora, A. (2009). Mantenimiento. México: AlfaOmega.

Muñoz, M. (2002). Mantenimiento Industrial. Universidad Carlos de la Madrid. 